\title{
Erratum: Health-related quality of life of antiretroviral treatment defaulters in Botswana
}

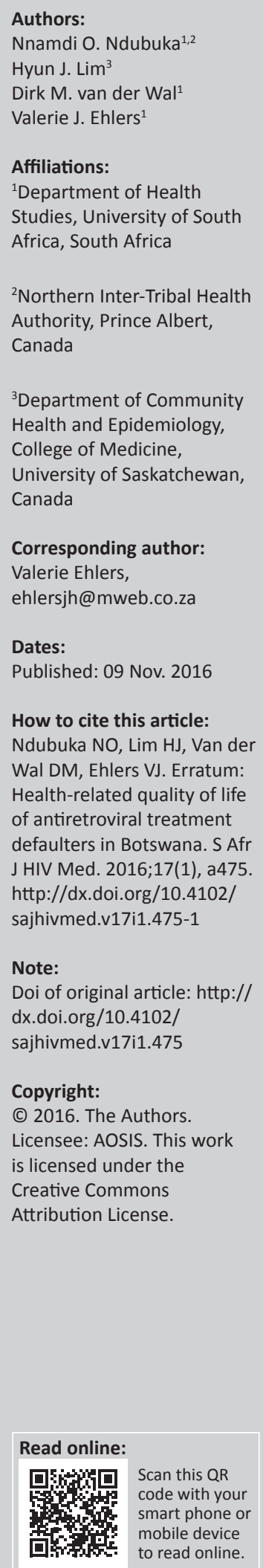

In the publication of the article, the first author's second affiliation country was published incorrectly as South Africa instead of Canada. This has been corrected under the affiliations sections of the erratum. 